\title{
MedienPädagogik
}

Zeitschrift für Theorie und Praxis der Medienbildung

Jahrbuch Medienpädagogik 3.

Zweitveröffentlichung aus: Jahrbuch Medienpädagogik 3. (2003) Opladen: Leske + Budrich. Herausgegeben von Ben Bachmair, Peter Diepold und Claudia de Witt.

\section{Kindliche Medien- und Werbekompetenz als Thema der Medienforschung ${ }^{1}$}

Dorothee M. Meister und Uwe Sander

\section{Kinder und Medienforschung}

Das Mediensystem ist heute in weiten Bereichen marktwirtschaftlich aufgebaut und richtet sich auch und zunehmend an Kinder als Kunden. Kinder sind damit eine wichtige Zielgruppe der Medien geworden. Das betrifft zum einen solche Medien, die wie z.B. Hörkassetten (fast) reine Kindermedien sind (vgl. Gangloff/Abarbanell 1994, S. 221-226; Treumann/Gartemann/Schnatmeyer/Röllecke 1996), aber auch alle anderen Medien wie Computer-Spiele), Video, Bücher und Zeitschriften/Hefte und natürlich das Fernsehen (vgl. Meister/Sander 1998; Erlinger 1997). Radikale Positionen sehen mit dieser Entwicklung eine (z.T. sehr negativ bewertete: Glogauer 1995) grundlegende Veränderung der Kindheit verbunden, die Rede ist etwa vom „Verschwinden der Kindheit“ (Postman 1983), vom „allmählichen Verschwinden der Wirklichkeit“ (von Hentig 1984) oder vom „Ende der Erziehung“ (Postman 1997). In der Medienforschung werden jedoch Jugendliche oder Erwachsene viel intensiver untersucht als Kinder, obgleich es selbstverständlich auch empirische Medienstudien gibt, die Kinder mit einbeziehen oder sich nur auf Kinder beziehen (vgl. Bachmair/Kress 1996, Charlton/Neumann-Braun u.a. 1995, Meister/Sander 1998, Baacke/Sander/Vollbrecht u.a. 1999). Ein Grund hierfür dürfte sein, dass Kinder-Medien-Studien, und vor allem Studien mit jüngeren Kindern, nicht auf die klassischen methodischen Erhebungsinstrumentarien (schriftliche Fragebogenbefragung, standardisierte mündliche oder Telefoninterviews etc.) der Medienforschung zurückgreifen können. So lassen sich z.B. 12jährige oder 14jährige durchaus mit einem Instrument befragen, das auch auf ältere Jugendliche oder Erwachsene angewendet werden kann. Bei jüngeren Kindern jedoch bilden Verständnisprobleme bei Fragen, schlechte bis nicht vorhandene Lese- und Schreibfähigkeiten, kaum entwickelte Zeitund Mengenvorstellungen und Konzentrationsprobleme große methodische Probleme. Mediennutzungsdaten müssen für diese Altersgruppe mühsam erhoben werden, so dass es nicht verwundert, dass die Stichprobe

1 Dieser Artikel stellt eine überarbeitete Fassung eines Beitrags dar aus: Heinzel, Friederike (Hg.): Methoden der Kindheitsforschung, Weinheim/München 2000. 
vieler Medienstudien erst mit den 14jährigen beginnt und ältere Personen einschließt.

\section{Medien-/Werbekompetenz - ein Konjunkturthema der Medienforschung}

„Medienkompetenz" hat sich in den letzten Jahren zu einer bedeutsamen pädagogischen Kategorie entwickelt, die sowohl die Pädagogik allgemein wie auch die Medienpädagogik speziell beschäftigt. Die Konjunktur dieses Begriffes resultiert zum einen aus den rasanten Entwicklungen im Bereich der Informations- und Kommunikationsmedien, die so gravierende gesellschaftliche Dynamiken in Gang gesetzt haben, dass heute vielfach von der Informations- bzw. zunehmend von der Wissensgesellschaft gesprochen wird (vgl. Stehr 1994; Willke 1997). In diesem Zusammenhang ist eine breite Diskussion darüber entstanden, wie die Mitglieder einer modernen Gesellschaft mit diesen Entwicklungen umgehen sollen. Es steht die Forderung im Raum, dass sich jeder im „Informations- und Kommunikationsdschungel“ der Medienentwicklungen zurechtfinden sollte bzw. aus unterschiedlichsten Gründen auch genötigt ist, aktiv an den medialen und kommunikativen Veränderungen teilzuhaben. „Medienkompetenz“ wird hierfür vielfach als Schlüsselbegriff gesehen (vgl. von Rein 1996). Dabei besteht einerseits weithin Einigkeit darüber, dass Medienkompetenz teilweise ein Resultat autodidaktischer Bemühungen ist und teilweise sogar en passant erworben wird, andererseits sind sich Pädagogen weithin einig, dass der Bewältigung von Anforderungen in der „Wissensgesellschaft“ mittels pädagogischer Förderung entsprochen werden kann. Während pädagogische Förderung und notwendige Sozialisationsleistungen in der Vergangenheit vor allem Kindern und Jugendlichen zugeschrieben wurde, sind mit dem Begriff der „Medienkompetenz“ indes explizit alle Altersgruppen angesprochen, womit die Diskussion auch für die Erwachsenenbildung an Relevanz erhält (vgl. Dewe/Sander 1996).

Neben diesem eher medien- und informationsorientierten Begründungszusammenhang für Medienkompetenz als einer bedeutsamen pädagogischen Kategorie finden sich zum anderen gerade im Kontext von Kindheit noch weitere Legitimationsfoci. Da eine bewahrpädagogische Haltung in Anbetracht der Omnipräsenz der Medien heute kaum noch praktizierbar und auch pädagogisch nicht mehr gewollt ist - die Lebenswelt der Kinder hat sich heute in eine „Medienwelt“ (Baacke/Sander/Vollbrecht 1991) verwandelt und kann nicht mehr in einen medienfreien Schonraum rückverwandelt werden, gehen (medien-)pädagogische Alternativen heute eher in Richtung handlungsorientierter und alltagsnaher Ansätze. Wenn Kinder nicht mehr umfassend vor den Medien (hier auch: vor Werbung) „geschützt“ werden können, so sollen sie befähigt werden, autonom und bewusst damit umzugehen. Diese Befähigung verspricht Medienkompetenz (vgl. zusammenfassend Meister/Sander 1998). Medienkompetente Kinder, so die Meinung oder Hoff- 
nung, sollen in der Lage sein, den Anforderungen der Mediengesellschaft zu entsprechen. Die Kinder sollen sich dort selbst behaupten können, wo ihnen Erwachsene keinen umfassenden Schutz mehr gewähren können. Eine solche Argumentation findet sich auch im Zusammenhang anderer Altersgruppen. So ist z.B. mit Medienkompetenz im Erwachsenenalter die Hoffnung verbunden, dass die Erwachsenen im Alltag und im Beruf mit der rasanten Innovationsgeschwindigkeit der neuen Medien Schritt halten können (vgl. Dewe/Sander 1996; Arnold 1991; Tietgens 1989).

Hier allerdings konzentrieren wir uns auch die Lebensphase Kindheit und im weiteren noch spezifischer auf den Umgang mit Werbung. Bevor im folgenden Kinder bis 13 Jahre im Focus stehen, soll zunächst eine begriffliche Klärung von „Medienkompetenz“ vorgenommen werden. Danach werden wir auf der Grundlage einer empirischen Unter-

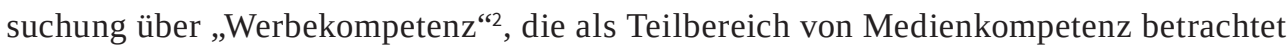
wird, entwickeln, auf welche Weise sich methodisch bei Kindern die Genese von Medienkompetenz nachzeichnen lässt.

\section{Medienkompetenz als wissenschaftliche Kategorie}

Als wissenschaftlicher Terminus wurde „Kompetenz“ - unter verschiedenen Zugängen und Voraussetzungen - vor allem durch die Arbeiten von Baacke, Bourdieu, Chomsky und Habermas (vgl. Baacke 1996; Habermas 1971; Chomsky 1972) bekannt. Der Kompetenzbegriff wurde von dem Linguisten Chomsky als eine im Mentalen verankerte Fähigkeit des Menschen angenommen, aufgrund eines immanenten Regelsystems eine potentiell unbegrenzte Anzahl von Sätzen zu erzeugen. Die Vorstellung einer „universellen Grammatik“ behauptet im Grunde, „dass alle Menschen potientiell über die Sprachmuster einer Universalsprache verfügen - und insofern gleich sind“ (Baacke 1996, S. 116). Die tatsächliche Erzeugung eines konkreten Satzes wird in diesem Modell mit dem Begriff der Performanz belegt. Wenn man sich wie Baacke auf Chomsky beruft, kann eine empirische Beobachtung vorhandener Medienkompetenz genauso wie der Ansatzpunkt pädagogischer Interventionen lediglich auf der Performanzebene liegen. Die Herausforderung für die Pädagogik besteht also im Spannungsverhältnis zwischen Kompetenz und Performanz und damit in der Nichtidentität eines universalen Regelsystems und regelgeleiteter aktueller Strukturierung in einer konkreten Sprechsituation. Baacke zieht aus dieser Differenz von Kompetenz und Performanz den Schluss, dass der ontologische Kompetenzbegriff auf ein Gleichheitspostulat hinausläuft, das in diesem Kontext auch pädagogische Förderung begründet, um die allen inhärente Kompetenz zur Performanz zu

2 Die hier vorgestellten Überlegungen basieren auf den Ergebnissen des Projektes „Kinder und Werbung“, das zwischen 1995 und 1997 an den Universitäten Bielefeld und Halle unter der Leitung von Baacke/Sander/ Vollbrecht u.a. (1999) durchgeführt wurde. 
bringen. Begründbar wird eine pädagogische Einflussnahme aus dem Umstand, dass die Performanzebene als empirisch wahrnehmbare Oberflächenstruktur menschlichen Handelns durch weitere Bedingungen wie subjektive Faktoren (Lebensgeschichte, Motivation) und gesellschaftlichen Variablen (institutionelle Bedingungen des Handelns, Rollenerwartungen an den Handelnden) genauso wie von situativen, entwicklungsbedingten, sozialen und kulturellen Variablen beeinflusst wird. Pädagogische Vermittlungsprozesse können insofern nicht auf der Kompetenzebene ansetzen, sondern zielen auf die Transformationsprozesse, mittels derer sich Kompetenz in Performanz realisiert. Insofern „geht es in impliziten Bildungsprozessen nicht um den Erwerb konkreter Handlungsmuster, sondern um den Erwerb von Strukturen“ (Dewe/Sander 1996, S. 129), da Kompetenz als kognitive Fähigkeit keine sachliche Phänomenebene, sondern die „Fähigkeit im Umgang mit Wissen selbst“ (ebd., S. 128) betrifft.

Wie deutlich geworden sein sollte, haben wir es also mit einem Dilemma zu tun, das darin besteht, dass Medienkompetenz lediglich auf der performativen Ebene beobachtbar ist, also nur auf dem handlungspraktischen Umgang mit Medien bzw. über die geäußerten Meinungen über Medien. Diese Beobachtung ist zudem angeleitet durch einen spezifischen medienpädagogischen Blick, der Anzeichen von Medienkompetenz bei den Kindern erwartet. Wird diese Erwartungshaltung durch Handlungsweisen oder Äußerungen der Kinder erfüllt, dann wird Medienkompetenz unterstellt. Wird diese Erwartungshaltung nicht erfüllt, dann führt die Beobachtung der Performanzebene dazu, ein Defizit auf der Kompetenzebene zu unterstellen. Bevor im weiteren auf dieses Verhältnis und auf Entwicklungsverläufe auf der performativen Ebene eingegangen wird, dienen einige Definition von Medienkompetenz zunächst der begrifflichen Klärung, wie von pädagogischer Seite aus Medienkompetenzen definiert und was entsprechend auf der Performanzebene erwartet wird.

Schorb (1995, S. 184ff.) beschreibt kommunikative Kompetenz als wichtiges Ziel zeitgemäßer Medienpädagogik. Seiner Ansicht nach wird kommunikative Kompetenz auf dem Wege handelnden Lernens erworben und umfasst - Habermas folgend - drei Bestandteile:

- eine analytische Komponente, die in der Fähigkeit zum Tragen kommt, in einem gemeinsamen Erkenntnisprozess die durch „Massenmedien“ vorgegebene Sichtweise zu durchbrechen, somit ihr Wesen aufzuhellen und gemeinsame Möglichkeiten zu ihrer Überwindung zu finden;

- eine kreative Komponente, die sich in der Fähigkeit realisiert, den herrschenden Kommunikationsstrukturen andere entgegenzusetzen, verbunden mit Strategien zur Vermittlung und zur Durchsetzung der eigenen gemeinsamen Interessen;

- eine kommunikative Komponente, die sich in der Fähigkeit der adäquaten, d.h. reflektierenden Erfahrungsbewältigung und -darstellung ausdrückt.

Baacke (1997; 1996) befasst sich mit dem Konzept der „Medienkompetenz“ im Kontext kommunikativer Kompetenz. Er leitet diesen Terminus aus einem 
„kulturellen In-der-Welt-Sein“ und einem gemeinsamen Wahrnehmungsbewältigungsprozess ab. Betont wird dabei die Tatsache, dass alle Menschen grundsätzlich mit der Fähigkeit ausgestattet sind, sich in der Welt erfolgreich und sozial zu bewegen, allerdings muß diese Ausstattung gefördert, zur Performanz gebracht werden. Dabei arbeitete Baacke folgende Teilbereiche dieser Medienkompetenz heraus:

Medien-Kritik befähigt das Individuum

- problematische gesellschaftliche Prozesse (z.B. Konzentrationsbewegungen) analysieren zu können;

- analytisches Wissen auf sich selbst und sein Handeln anwenden zu können;

- analytisches Denken und reflexiven Rückbezug sozial zu verantworten und abzustimmen.

Medien-Kunde (Wissen über heutige Mediensysteme) beinhaltet

- eine informative Dimension (klassische Wissensbestände wie „was ist ein duales Rundfunksystem..." etc.);

- eine instrumentell-qualifikatorische Dimension (d.h. Fähigkeit, die neuen Geräte auch bedienen zu können);

- Medien-Nutzung (als vorrangig rezeptive Anwendung von Medien oder als ein interaktives Angebot);

Medien-Gestaltung kann verstanden werden

- innovativ (im Sinne von Veränderungen/Weiterentwicklungen des Mediensystems) bzw.

- kreativ (als ästhetische Varianten, als ein Über-die-Grenzen der Kommunikationsroutine-Gehen) .

Medienkompetenz „meint also grundlegend nichts anderes als die Fähigkeit, in die Welt aktiv aneignender Weise auch alle Arten von Medien für das Kommunikations- und Handlungsrepertoire von Menschen einzusetzen.“ (Baacke 1996, S. 119). Aufenanger (1996) differenziert zunächst zwischen „Medienkompetenz“ und „medienpädagogischer Kompetenz“. Der erstgenannte Begriff zielt dabei auf die Mediennutzer; „medienpädagogische Kompetenz“ wird vor allem als relevante Zielgröße für Eltern, Lehrer, Erzieher usw. angesehen. Positionen von Baacke und Buschmeyer (1995) berücksichtigend, arbeitet Aufenanger (1996, S. 461) folgende Komponenten der komplexen Fähigkeit „Medienkompetenz", heraus:

- Wissen, bezogen auf Medien und ihre Anwendung;

- Verstehen von medialen Texten;

- Beurteilen von Medienangeboten und Mediensystemen;

- Genießen von Medienangeboten unter Abwägung anderer Handlungsalternativen; 
- Handeln mit und in bezug auf Medien als Informationsvermittler und zur Ausdrucksgestaltung.

Die genannten Definitionen verdeutlichen, dass Medienkompetenzen allgemeine Anforderungen darstellen, die Kompetenz- und Performanzelemente enthalten, und insofern versuchen, allgemeine Kriterien mit spezifischen Erwartungen in modernen Gesellschaften zu verbinden. Aufgrund der Vielfalt des Mediensystems muss geschlussfolgert werden, dass die Konkretion von Medienkompetenz in den verschiedenen Teilbereichen jeweils spezifisch realisiert werden muss. So lassen sich z.B. aus dem Vergleich „Kunst“ oder allgemeiner „Unterhaltung“ und „Werbung“ Unterschiede festmachen. Während „Unterhaltung“ in erster Linie Verstehen und Genus anspricht, erfordert eine „kompetente“ Rezeption von „Werbung“ vorrangig Wissen, (kritisches) Beurteilen und die Berücksichtigung, dass mit Werbung „Interessen“ verbunden sind.

Insofern erscheint es durchaus sinnvoll zu sein, bei empirischen Untersuchungen des Gegenstandes „Medienkompetenz“ zunächst Teilbereiche genauer zu untersuchen, um so zu empirisch gesättigten Aussagen des Gesamtzusammenhangs gelangen zu können. Eine solche Konkretion von Medienkompetenz bei älteren Kindern soll im folgenden am Beispiel von „Werbekompetenz“ vorgenommen werden.

Datengeleitete These: Die „vorkritischen Genese“ von Werbekompetenz bei Kindern In dem Forschungsprojekt „Kinder und Werbung“ haben wir versucht, den Aspekt der Werbekompetenz, als einen Teilbereich von Medienkompetenz, bei Kindern empirisch zu ermitteln. ${ }^{3}$ Bei Werbung legt unseres Erachtens ein medienpädagogisches Verständnis von Werbekompetenz nahe, neben der Eigenperspektive auch die verschleiernden, manipulativen Absichten der Werbung zu erkennen. Kompetenter Umgang mit Werbung erfordert demnach auch das Durchschauen der Interessen von Werbetreibenden und ist insofern anschlussfähig an die vorgenannten Definitionen. In Baackes Terminologie wäre Werbekompetenz vor allem auf der Ebene der Medienkritik und der Medienkunde anzusiedeln.

„Werbekompetent“ in unserer Definition ist jemand, der neben Wissen über Werbung auch die Interessenhintergründe von Werbung berücksichtigen kann. Dazu muss die Person fähig sein, seine eigene Perspektive auszutauschen und die Perspektive Dritter einzunehmen. Insofern kann „Werbekompetenz“ - die wir als Teilmenge von Medienkompetenz charakterisiert haben - als Entwicklungs- und Sozialisationsaufgabe aufgefasst werden, die jedes Individuum - mit seinen Möglichkeiten und auf seine Weise - in Sozialisati-

3 Vgl. hierzu insbesondere die Ausführungen von Böhm-Kasper/Fischer sowie Meister in dem Band Baacke/ Sander/Vollbrecht u.a. (1999). 
onsprozessen zu erbringen hat, und bei der es pädagogisch ermutigt, begleitet und unterstützt werden kann und soll.

Als methodologische Grundlage kann u.a. auch der Struktur genetische Ansatz von Piaget (1976) herangezogen werden. Dieser erlaubt, insbesondere Entwicklung und Veränderung in der Perspektiven- bzw. Rollenübernahme als Aspekt der sozialen Kognition differenziert zu betrachten. Entscheidend für eine entwickelte Werbekompetenz wären demnach vor allem die Herausbildung der Fähigkeit zur Dezentrierung, die Zurückdrängung der Ich-Aufdringlichkeit bei Urteilsbildungen und die Fähigkeit der gleichzeitigen Koordination mehrerer Dimensionen.

Ohne nun auf Details der Ergebnisse unserer quantitativen und qualitativen Studie einzugehen, sollen im folgenden drei Aspekte betrachtet werden, die für Kinder bis 13 Jahren von Bedeutung sind. In der Studie wurden 5- bis 13jährige Kinder befragt. Da aber eine These vertreten wird, die einer inhärenten Entwicklungslogik folgt, sind die Überlegungen auch für ältere Altersgruppen relevant. Die generelle These lautet, dass Werbekompetenz als universale Kategorie im Sinne von Medienkompetenz mit zunehmendem Alter und Bildungsgrad zunimmt, sie entwickelt sich bei Kindern jedoch zunächst in einer „,vorkritischen“ Form. Diese These wird in drei Schritten begründet, zum einen hinsichtlich der quantitativen Ergebnisse in bezug auf eine sich entwickelnde Werbekompetenz, zum zweiten - qualitativ begründet - hinsichtlich moralischer Bewertungen und sozialen Beziehungen bei der Beurteilung von Werbung sowie hinsichtlich selbstkritischer Reflexionen vom eigenen Umworbensein.

Aussage 1: Formal gesehen werden Kinder umso werbekompetenter, je älter und gebildeter sie sind.

In unserer quantitativen Untersuchung ${ }^{4}$ wurde das Konstrukt der Werbekompetenz in Hinblick auf Kinder operationalisiert. Ausgehend vom rollenabhängigen „Interesse an Werbung“ wurden zur empirischen Erforschung der Werbekompetenz den Kindern folgende Fragen gestellt:

Woher kennst Du Werbung?

Wie gefällt Dir Werbung allgemein?

Glaubst Du, was in der Werbung gesagt wird?

Was glaubst Du, warum wird Werbung gemacht?

Schon bei der Frage, woher Kinder Werbung kennen, war ein Alters- und Bildungseffekt erkennbar. Während Grundschulkinder fast ausschließlich das Fernsehen als Werbeträger nennen, können Gymnasiasten eine Vielzahl von Werbeträgern benennen.

4 Insgesamt wurden 1600 Kinder im Alter zwischen 6 und 13 Jahren in der Region Halle und der Region Bielefeld befragt (vgl. zu den quantitativen Ergebnissen im einzelnen: Baacke/Sander/Vollbrecht u.a. 1999). 
Die Untersuchung der generellen Akzeptanz von Werbung durch Kinder erfolgte durch die Frage „Wie gefällt Dir Werbung allgemein?“. Die Kinder waren bei dieser Frage aufgefordert, Schulnoten von 1 bis 6 zu vergeben. Bei den Ergebnissen zeigt sich, dass jüngere Kinder Werbung in der Mehrheit positiv bewerten (62,7\% der 6jährigen vergaben die Noten 1 und 2, während dies bei den 12-13jährigen nur noch 22,8\% waren). Mit zunehmendem Alter wird Werbung von den befragten Kindern überwiegend negativ beurteilt. In der Alterskohorte der 11-13jährigen stellen die Gymnasiasten die Gruppe mit den am häufigsten geäußerten Ablehnungen gegenüber Werbung.

Ein weiterer Aspekt des kompetenten kindlichen Umganges mit Werbebotschaften wurde mit der Frage „Was glaubst du, warum wird Werbung gemacht?“ erhoben. Die Frage zielt im wesentlichen auf den Kenntnisaspekt, vor allem Sachkenntnisse sind gefordert. Diese Sachkenntnisse weisen jeweils einen individuell unterschiedlichen Grad an Allgemeinheit, Systemhaftigkeit, Anschaulichkeit, Disponibilität und Sinnhaftigkeit auf. Zur Beantwortung sollten die Kinder mit „ja“ oder „nein“ über die Angemessenheit nachstehender Vorgaben entscheiden:

1 Damit Deine Eltern wissen, was sie Dir kaufen können?

2 Damit Du weißt, was es Neues gibt?

3 Damit neue Sachen schnell bekannt werden?

4 Damit sich Leute Dinge kaufen, die sie gar nicht brauchen?

5 Weil TV, Radio, Zeitschriften damit Geld verdienen?

6 Damit die Firmen mehr verkaufen

7 Damit Kinder was zu lachen haben?

8 Damit man zwischendurch etwas anderes machen kann?

9 Damit das Fernsehen nicht so langweilig ist?

Die vorgegebenen Antworten über die Gründe/Ursachen/Zwecke von Werbung lassen sich dabei in folgende drei Bereiche einteilen,

- Bereich der informationellen und/oder affektive Beeinflussung der Verbraucher (Fragen 1-3):

- Bereich der Hauptursachen/des Wesens von Werbung in der Marktwirtschaft (Fragen 4-6);

- Bereich zufälliger, nebensächlicher, oberflächlicher Erscheinungen bzw. Transformationen beobachteter oder eigener Verhaltensweisen (Fragen 7-9).

Die Bedeutung der Fragestellung läuft sowohl auf die Qualität eines Systems von Kenntnissen bezüglich der Funktion von Werbung in der Marktwirtschaft als auch auf die Fähigkeit hinaus, Erscheinung und Wesen, Notwendiges und Zufälliges voneinander zu unterscheiden (Verknüpfung von Kenntnissen und Fähigkeiten als Könnensqualität). Die Ursachen für die Ausprägung dieses Könnens liegen sowohl im Bereich der Kenntnisvermittlung, d.h. der ständigen Erweiterung des Systems von Kenntnissen, als auch in altersspezifischen Besonderheiten (Denkentwicklung usw.).

Die Antworten der untersuchten Kinder weisen darauf hin, dass die Mehrheit der befragten Kinder - selbst im Grundschulalter - weiß, dass Wer- 
bung ein Mittel von Firmen ist, um Produkte erfolgreich zu vermarkten (77,6\%). Weiterhin kann ein Großteil der Kinder nachvollziehen, dass sich Radio, Fernsehen und Printmedien durch Werbeeinnahmen finanzieren (73,3\%). Analysiert man jedoch die Antwortbereiche 7 bis 9, so wird deutlich, dass insbesondere Grundschulkinder die ökonomischen Ziele des Werbemarktes nicht eindeutig durchschauen:

\begin{tabular}{llllllll}
\hline \multicolumn{2}{l}{$\begin{array}{l}\text { Was glaubst Du, warum wird Werbung } \\
\text { gemacht? }\end{array}$} & \multicolumn{7}{l}{ Alter in Jahren } \\
\hline \multirow{2}{*}{$\begin{array}{l}\text { 7) Damit Kinder was zu lachen } \\
\text { haben? }\end{array}$} & ja & 34,6 & 53,0 & 53,1 & 20,7 & 21,0 & 24,7 \\
& nein & 59,2 & 38,1 & 41,0 & 75,1 & 72,5 & 69,4 \\
& w.n. & 6,2 & 8,9 & 5,9 & 4,3 & 6,5 & 5,9 \\
8) Damit man zwischendurch & ja & 47,8 & 53,1 & 52,5 & 44,4 & 43,9 & 45,5 \\
etwas anderes machen kann? & nein & 45,9 & 37,1 & 40,1 & 53,2 & 48,6 & 50,5 \\
& w. n. & 6,3 & 9,8 & 7,4 & 2,4 & 7,5 & 4,0 \\
9) Damit Fernsehen nicht so & ja & 26,1 & 42,5 & 39,8 & 16,6 & 16,1 & 14,5 \\
langweilig ist? & nein & 68,4 & 46,6 & 53,1 & 81,0 & 80,3 & 81,0 \\
& w.n. & 5,5 & 10,9 & 7,1 & 2,4 & 3,6 & 4,5 \\
\hline
\end{tabular}

Tab. 1.: Bereich zufälliger, nebensächlicher, oberflächlicher Erscheinungen bzw. Transformationen beobachteter oder eigener Verhaltensweisen (7-9); w.n. = weiß nicht.

Die Beantwortung dieser Fragen zeigt, dass für jüngere Kinder der Unterhaltungswert von Werbung im Vordergrund steht. Des weiteren geht augenscheinlich das persönliche Verhalten beim Fernsehkonsum in die Beantwortung ein (insbesondere Antwortmöglichkeit 8).

Die Antwortmöglichkeiten 1 bis 9 wurden zu einem Index zusammengefasst und daraus eine neue Variable $\left(\mathrm{F}_{\Sigma}\right)$ gebildet. Diese Variable weist als minimalen Wert „0“ und als maximalen Wert „9“ auf; für eine ,zutreffende“ Antwort der 9 Fragen erhält die Variable jeweils den Wert 1, so dass maximal 9 Punkte möglich sind. Der Mittelwert des Index ist mit 5,82 Punkten relativ hoch. 58,2\% der Kinder weisen eine Punktzahl höher 5 auf; von dieser Gruppe erreichte über 1/3 der Kinder sogar 6 und mehr Punkte. Es zeigt sich somit auch mit dieser künstlich gebildeten Variablen, dass die befragten Kinder zu einem großen Teil die Funktion von Werbung kennen, um die Hintergründe von Werbemechanismen (wenn auch plakativ) wissen und mit zunehmendem Alter den Perspektivenwechsel zwischen eigenem Verhalten und Werbeintention vornehmen können.

Die Ergebnisse zeigen zum einen, dass das Geschlecht und die Ost-West-Zugehörigkeit keinen signifikanten Einfluss auf die Beantwortung der von uns gestellten Fragen ausüben. Dieses Ergebnis scheint aus sozialisationstheoretischer Sicht plausibel, da Mädchen und Jungen genauso wie Kinder in Ost- und Westdeutschland gleichermaßen mit Werbung konfrontiert werden und somit die gleichen gesellschaftlich akzeptierten Deutungsmuster für die Intention der Werbung entwickeln. 
Signifikante Mittelwertunterschiede zeigen sich hingegen im Alter und der Schulzugehörigkeit. Das Alter als unabhängige Variable zeigt einen hochsignifikanten Zusammenhang mit dem neu konstruierten Index. Auch hinsichtlich des Schultyps zeigen sich signifikante Mittelwertsunterschiede. Diese wurden für die (weiterführenden) Schulen bei konstantem Alter ermittelt, um eine Drittvariablenverzerrung auszuschließen. Hauptschüler weisen demnach den niedrigsten Mittelwert auf $(5,68)$, während Realschüler $(6,73)$ und Gymnasiasten $(7,04)$ höhere Werte erreichen.

\begin{tabular}{cc}
\hline Alter in Jahren & Mittelwert der Variablen $\mathrm{F}_{\Sigma}$ \\
\hline 6 & 4,34 \\
7 & 4,75 \\
8 & 5,03 \\
9 & 5,57 \\
10 & 6,69 \\
11 & 6,53 \\
12 & 6,53 \\
\hline
\end{tabular}

Tab. 2.: Mittelwertunterschiede in der Variablen $\mathrm{F}_{\Sigma}$ nach Alter.

Aufgrund der Ergebnisse lässt sich also zunächst der Schluss ziehen, dass das Alter und die unter dem Begriff „Schulbildung“ subsumierten sozialen und kognitiven Dispositionen der untersuchten Kinder wesentliche Faktoren darstellen, die Einfluss auf die Ausbildung einer kindlicher Medien- bzw. Werbekompetenz haben.

Aus dieser Aussage heraus den Schluss zu ziehen, dass sich mit zunehmendem Alter eine Medien- und Werbekompetenz sozusagen „en passant“ entwickelt, wird den Ergebnissen allerdings nicht gerecht. Vielmehr weisen die Daten darauf hin, dass z.T. auch ältere Kinder die Intention der Werbung nicht ohne weiteres durchschauen können. So wussten von den 11-13jährigen immerhin über 13\% nicht, dass Fernsehen, Radio und Zeitschriften mit Werbung Geld verdienen. Auch das Ergebnis, dass insbesondere Hauptschüler Schwierigkeiten haben, die Intention von Werbung zu durchschauen lässt darauf schließen, dass nicht generell von „Werbekompetenz“ in diesem Alter gesprochen werden kann. Dass es sich zudem meist sogar um eine „,vorkritische“ Variante handelt, darauf verweisen auch die quantitativen Daten. Zwar nimmt äußerlich gesehen mit zunehmendem Alter die Werbekritik zu und die Glaubwürdigkeit von Werbung ab, der Referenzrahmen für die Begründung der Ablehnung scheint allerdings weniger universeller Art zu sein, sondern bezieht sich eng auf den sozialen Kontext. So weisen 90,5\% aller Kinder, die Werbung ablehnen darauf hin, dass ihr Vater Werbung ebenso ablehnt, bei den 11-14jährigen sind dies 96,2\%. Wir interpretieren diese Daten in die Richtung, dass die Kinder zunehmend den Erwartungen, die im Elternhaus und in ihrer Gleichaltrigengruppe im Hinblick auf Werbung existieren, gerecht werden und diese reproduzieren. Dies muss noch nicht bedeuten, dass sie auch ein reflexives und strukturales Verständnis des Komplexes Werbung entwickelt haben. 
Auf die These einer „vorkritischen Werbekompetenz“ soll im weiteren aufgrund der qualitativen Ergebnisse vertiefend eingegangen werden. Dabei wird insbesondere auf moralische Bewertungen und soziale Bezüge sowie die Differenz von Einstellung und Verhalten in bezug auf Werbung eingegangen.

Aussage 2: Zur Bedeutung moralischer Bewertungen und enger sozialer Beziehungen bei der Beurteilung von Werbung

Genauso wie beim quantitativen Erhebungsteil zeigt sich bei den qualitativen Ergebnissen $^{5}$ unserer Befragung (vgl. Baacke/Sander/Vollbrecht u.a. 1999), dass das Hintergrundwissen über Werbung zunimmt und Werbung allgemein von den Kindern mit zunehmendem Alter kritisch hinterfragt wird, was ein Indiz für steigende Werbekompetenz resp. Medien-Kritikfähigkeit sein kann. Auffällig erweist sich indes, dass bei den 11- bis 12jährigen moralische Aspekte bei der Beurteilung von Werbung eine große Rolle spielen, das heißt, sie achten bei der Einschätzung von Werbungen in hohem Maße auf den Wahrheitsaspekt. Es geht also darum, ob proklamierte Ziele zum einen richtig und zum anderen glaubwürdig sind. Wenn Kinder den Eindruck gewinnen, moralische Standards würden verletzt, stößt Werbung auf Ablehnung bei ihnen.

Trotz einer generellen kritischen Einstellung variiert die Beurteilung einzelner Werbespots im Einzelfall jedoch sehr stark und kann auch positiv ausfallen. In ihrer generell kritischen Haltung zur Werbung fühlen sich die Kinder dabei zumeist von den Eltern und auch von Freunden unterstützt. Immer wieder ziehen Kinder die Familie quasi als Autorität im Hintergrund bei ihrer Einschätzung heran, wodurch ein hoher Einfluss und Vorbildcharakter der Eltern zum Ausdruck kommt. Bei denjenigen Kindern, die der Werbung eher kritisch gegenüberstehen, besteht überwiegend ein familialer Konsens in dieser Frage. Trotzdem fühlen sich Kinder angesprochen von Werbung. Teilweise wird die Begründung, warum bestimmte Werbungen den Kindern trotz genereller Kritik im Einzelfall gefallen, über eine einheitliche Ausnahmemeinung in der Familie hergeleitet. Der Orientierungsrahmen der Kinder bewegt sich also in hohem Maße darauf, was gut und richtig ist und was nicht, welches Verhalten, welche Werte und Orientierungen glaubwürdig oder nicht sind. Die moralischen Ansprüche fallen in diesem Alter teilweise sehr kategorial aus, da sie geprägt sind von der Suche nach eindeutigen Orientierungen und Identifikationen, wie dies bei der 11jährigen Interviewpartnerin Ulrike deutlich wird:

I: Und wie ist denn das, gefällt Dir (bezieht sich auf Zeitschriften und andere Werbeträger) Werbung oder findest Du das langweilig?

5 Insgesamt wurden 33 Interviews mit Kindern im Alter von fünf bis zwölf Jahren zwischen Dezember 1995 und Februar 1996 geführt, darunter 19 Mädchen und 14 Jungen aus Bielefeld und Halle an der Saale. 
U: Na, das finde ich dumm, für Zigaretten zu ... Das ist immer neue Werbung und so, das ist ... Aber vielleicht für Unicef oder so kann man ja Werbung machen. Für so was sinnvolles, also für Unicef, dass man da was spendet, oder eben für solche Tiere. Das ist dann auch nur Werbung gegen Tierversuche und so. Aber, dass man da mal irgendwas spendet oder so. Ich finde so was muss man machen, aber wenn man da irgendwie für Zigaretten ... so was nicht.

Wenn Kinder die gezeigten Werbungen und die damit verbundenen Produkte für glaubwürdig und gut befinden oder die Werbung witzig ist und Überraschungseffekte beinhaltet, sind sie durchaus bereit, diese Produkte zu vertreten, teilweise wären die Kinder dann sogar bereit, für diese zu werben. Die quantitativen Ergebnisse sowie die Analyse der qualitativen Interviews lassen uns zu der Annahme kommen, dass die Einstellung zur Werbung zunächst als Übernahme moralischer Stereotype erfolgt. Eine individuelle bzw. universale Begründungsfähigkeit im Sinne einer Reflexion des Sachverhaltes ist allerdings meist noch nicht entwickelt, sondern zeigt sich zunächst lediglich in Form einer „Als-ob-Struktur“.

Aussage 3: Eine selbstkritische Reflexion vom eigenen Umworbensein ist wenig ausgeprägt

Die Ergebnisse insbesondere der qualitativen Interviews weisen darauf hin, dass Werbekritik zwar auf der Einstellungsebene geäußert wird, dass aber die aus der Psychologie bekannte Trennung von Einstellung und Verhalten vorhanden ist. Dies zeigt sich auch darin, dass keine ausgeprägte Reflexion vom eigenen Umworbensein gegeben ist.

Gerade wenn Kinder Konsum gegenüber aufgeschlossen sind und/oder ein spezifisches Interesse wie Sport oder Musik haben, fühlen sie sich durch die Werbung informiert und schätzen Werbung dann in hohem Maße. Wenn sich Kinder informiert fühlen, beispielsweise von Trailern für spätere Filme oder bei Werbungen für Musik usw., haben sie nicht den Eindruck, umworben zu sein, sondern hilfreiches Neues zu erfahren, um sich besser orientieren zu können. Kritik wird in solchen Feldern des eigenen Interesses dann ausgeklammert, und die Kinder sind für die vermittelten Botschaften umso empfänglicher. Dies gilt auch, wenn die Kinder bestimmte Sendeformen wie Ratespiele präferieren. Gerade in den privaten Programmen werden solche Sendeformen in Form von Werbefilmen dargeboten, bspw. die Sendung „Glücksrad“. Wenn Kinder diese Darbietungsformen schätzen, fühlen sie sich kaum als Umworbene, sondern sehen in erster Linie ihren Spaß am Mitraten.

Obwohl Kinder zwischen 10 und 12 Jahren schon recht genau benennen können, warum Werbung und mit welcher Absicht sie gemacht ist, beziehen sie dieses Wissen kaum auf sich selbst, womit es abstrakt bleibt. Eine Absicht können sie Werbung im Grunde nur unterstellen, wenn sie sich nicht persönlich angesprochen fühlen. Viele Kinder nehmen sich zwar auch als Umwor- 
bene von der Werbeindustrie wahr und zeigen zudem ein ausgeprägtes Konsumentenverhalten. Darüber hinaus verfügen sie über Erfahrung mit Konsum und Merchandisingprodukten und haben nicht nur im Spielzeugbereich bereits, wechselnde Vorlieben und Moden entwickelt. Die eher kritische Haltung Werbung gegenüber auf der Verhaltensebene bedeutet also nicht, dass sie sich von Werbung auf der Handlungsebene nicht beeinflussen lassen.

Dies hängt auch damit zusammen, dass sie zwar die Absicht von Werbung kennen; Hintergrundinformationen, wer Werbung veranlasst und warum sie gemacht wird, sind allerdings nur rudimentär vorhanden. So bezweifeln beispielsweise viele Kinder die Sinnhaftigkeit von Werbung allgemein. Begründet wird dieses Unverständnis häufig damit, dass in der Familie ja sowieso eingekauft wird und für die täglichen Dinge des Alltags für sie kein Informationsbedarf besteht. In der Vorstellung der Kinder sind die eigenen Konsumwünsche im Allgemeinen dann auch eher am Preis orientiert und richten sich darüber hinaus noch sehr häufig nach dem Geschmack der Eltern, oder, in den Worten der 11jährigen Lisa ausgedrückt: „Ich kauf nix, was meinem Eltern doof finden, weil, das find' ich auch doof, meistens".

Eine hohe Beeinflussbarkeit scheint auch aufgrund der kritischen Grundhaltung Werbung gegenüber gegeben zu sein, die als Paradoxie der „kritischen Haltung“ bezeichnet werden kann. Gerade die bei Kindern so einheitlich abwertende Haltungen speziellen Werbungen gegenüber (z.B. Waschmittelwerbung), überzeugt sie davon, dass Werbung im Grunde nicht stimmt. Es sind vor allem die moralischen Wertungen, dass in Werbungen bspw. die Unwahrheit gesagt wird, die zumindest für bestimmte Bereiche Ablehnungen her vorrufen. Dies veranlasst sie zu dem Schluss, aufgrund mangelnder Glaubhaftigkeit der Werbungen selbst nicht beeinflussbar zu sein. Ablehnung wird automatisch mit Nicht-Wirkung in Verbindung gebracht. Dadurch geraten aber alle Formen indirekter Wirkungen oder der Einfluss durch Bekanntheit aus dem Blick und werden negiert.

Die Kinder gestehen dann zwar häufig offen ein, selbst schon einmal auf Werbung hereingefallen zu sein, diese Erfahrungen in Kombination, dass Werbung häufig nicht stimmt, erweckt bei ihnen indes den Eindruck, im Grunde inzwischen doch recht unabhängig von Werbemaßnahmen Kaufentscheidungen zu treffen, wenngleich ein Einfluss bei anderen Personen nicht ausgeschlossen wird. Die von ihnen selbst konstatierten Kaufwünsche aufgrund von Werbung definieren sie meist als Information, um über die Produkte der eigenen Individualität Ausdruck zu verleihen.

Teilhabe am Konsum und Partizipation an der Warenwelt können dann problemlos von den Kindern in ihre Vorstellungsweit integriert werden, ohne dem Eindruck zu unterliegen, sich allzu sehr von der Werbewelt beeinflussen zu lassen. Die Ausmaße des Einflusses variieren indes: Bei einem hohen Interesse für Sport (in dieser Altersgruppe sind dies vor allem Jungen) ist die Nähe zu Markenkleidung besonders hoch. Sportclubs oder einzelne Sportler stellen heute immer auch Werbeträger dar und sie werben insbesondere für spezielle Sportmarken. Über das Sportinteresse findet somit bei den Kindern 
automatisch eine Heranführung an bestimmte Marken statt, die zudem in den Peergroups einen bestimmten Status und Lebensstil, der über Kleidung symbolisiert wird, generiert. So hält beispielsweise der 11jährige Maurice die meiste Werbung für langweilig, die Werbung, die seinen Interessen, nämlich Sport gilt, empfindet er jedoch als informativ und orientiert sich durch zusätzliche Besuche von Warenhäusern über die neuesten Trends. Er definiert dann Markenkleidung nicht als Werbemaßnahme“, sondern hebt vor allem die „Qualitätsaspekte“ von bestimmten Artikeln hervor. Seine Wahl von Produkten sieht er als individuellen Stil an und blendet in diesem Bereich die Effektivität von Werbestrategien vollkommen aus.

Auch diejenigen Gruppen, die Werbung prinzipiell ablehnen, lassen sich von Werbestrategien ansprechen. Dies geschieht insbesondere dann, wenn Interessen für bestimmte Themen oder Musikrichtungen vorhanden sind oder sogar ein ausgesprochenes „Fanverhalten“ vorhanden ist. In diesen Fällen definieren diese Gruppen ihr Konsumverhalten dann nicht als von Werbung beeinflusst, sondern diese Gruppen heben ihren speziellen Lebensstil hervor, der dann allerdings eine ganz bestimmte Konsumhaltung bis hin zum Kauf von Merchandisingprodukten mit sich bringt.

\section{4. $\quad$ Fazit}

Kinder im Alter zwischen 10 und 13 Jahren kennen sich im Werbebereich gut aus, sie wissen auf einer abstrakten Ebene zum Großteil um Werbeintentionen und Werbemechanismen und nehmen überwiegend eine kritischen Grundhaltung gegenüber Werbung ein, auch wenn einzelne Werbungen unter ästhetischen, humoresken oder inhaltlichen Gründen sehr geschätzt werden. Werbung „wirkt“ in der Altersgruppe aber durchgängig, trotz unterschiedlicher vorfindbarer Einstellung zur Werbung. Auch wenn sich in der Gruppe der älteren Kinder differente Typen (wie Werbekritiker, Konsumorientierte, Fans etc.) entwickeln, kann sich Werbung doch bei allen Typen durchsetzen, allerdings über verschiedenste Mechanismen. Deutlich geworden sollte sein, dass die Kinder auf einer vordergründigen Weise (auf der Performanzebene) den vermeintlichen Erwartungen einer „kritischen“ Haltung entsprechen.

Kinder dieses Alters übernehmen also zunächst in einer Art „Als-ob-Struktur“ die Meinungen über Werbungen in ihrer Umgebung. Schwierigkeiten haben sie indes häufig noch damit, mit dem abstrakten Wissen selbstreflexiv umzugehen, die Ich-Aufdringlichkeit bei der Urteilsbildung zurückzudrängen und gleichzeitig mehrere Perspektiven zu koordinieren. Insofern handelt es sich in diesem Alter noch um eine „vorkritische“ Werbekompetenz. Insgesamt wird auch in diesem Alter deutlich, dass sich Werbekompetenz nicht automatisch ergibt, sondern von vielfältigen Umgebungen, die Kinder zunächst Argumentationsfolien liefern, unterstützt wird. Erst über die Auseinandersetzung mit ihrer eigenen Perspektive und der von Bezugspersonen können sie zu einer generalisierten Perspektive gelangen, und damit 
Werbe- bzw. Medienkompetenz erhalten, indem sie nämlich auch verallgemeinerte Perspektiven auf sich selbst reflektieren können.

\section{Literatur}

Arnold, R.: Betriebliche Weiterbildung. Bad Heilbrunn 1991.

Aufenanger, St.: Zur Zusammenarbeit von Elternhaus, Schule und Hochschule in der Medienerziehung. In: Pädagogik und Schulalltag, 4/1996, S. 460-470.

Baacke, D.: Medienkompetenz - Begrifflichkeit und sozialer Wandel. In: Rein, A. von (Hrsg.): Medienkompetenz als Schlüsselbegriff. Bad Heilbrunn 1996, S. 112-124.

Baacke, D.: Medienpädagogik. Tübingen 1997

Baacke, D./Sander, U./Vollbrecht, R.: Medienwelten Jugendlicher. Opladen 1991.

Baacke, D./Sander, U./Vollbrecht, R. u.a. (Hrsg.): Zielgruppe Kind. Kindliche Lebenswelten und Werbeinszenierungen. Opladen 1999.

Bachmair, B./Kress, G. (Hrsg.): Höllen-Inszenierung „Wrestling“. Beiträge zur pädagogischen Genre-Forschung. Opladen 1996.

Böhm-Kasper, 0./Fischer, A.: Überlegungen zum Konzept „Entwicklung von Werbekompetenz“. In: Baacke, D./Sander, U./Vollbrecht, R. u.a. (Hrsg.): Zielgruppe Kind: Kindliche Lebenswelten und Werbeinszenierungen. Weinheim/München 1999 (im Erscheinen).

Buschmeyer, H.: Pädagogische Überlegungen zum Konzept „Medienkompetenz“. In: Informationen Weiterbildung Nordrhein-Westfalen, Heft 5/1995, S. 20-24.

Charlton, M./Neumann-Braun u.a.: Fernsehwerbung und Kinder. 2 Bde. Opladen 1995.

Chomsky, N.: Aspekte der Syntax-Theorie. Frankfurt/M. 1972.

Dewe, B./Sander, U.: Medienkompetenz und Erwachsenenbildung. In: Rein, A. von (Hrsg.): Medienkompetenz als Schlüsselbegriff. Bad Heilbrunn 1996, S. 125-142.

Erlinger, H.D. (Hrsg.): Kinder und der Medienmarkt der neunziger Jahre. Opladen 1997.

Gangloff, T.P./Abarbanell, S. (Hrsg.): Fernsehen in Deutschland, Hamburg/Stuttgart 1994.

Glogauer, W.: Die neuen Medien verändern die Kindheit. Nutzung und Auswirkungen des Fernsehens, der Videofilme, Computer- und Videospiel, der Werbung und Musikvideoclips. Weinheim 1995.

Habermas, J.: Vorbereitende Bemerkungen zu einer Theorie der kommunikativen Kompetenz. In: Habermas,J./Luhmann, N.: Theorie der Gesellschaft oder Sozialtechnologie. Frankfurt 1971.

Hentig, H. von: Das allmähliche Verschwinden der Wirklichkeit. Ein Pädagoge ermutigt zum Nachdenken über die neuen Medien. München/Wien 1984.

Meister, D. M.: Zur Genese von Werbekompetenz beTKinder. Auswertungen der qualitativen Interviews mit Kindern zum Thema Werbung. In: Baacke, D./Sander, U./Vollbrecht, R. u.a. (Hrsg.): Zielgruppe Kind: Kindliche Lebenswelten und Werbeinszenierungen. Weinheim/München 1999.

Meister, D./Sander, U.: Kindheit und Jugend in der Mediengesellschaft. Bereichsrezension. In: Sozialwissenschaftliche Literaturrundschau 1/1998, S. 5-16.

Piaget, J.: Die Äquilibration der kognitiven Strukturen. Stuttgart 1976.

Postman, N.: Das Verschwinden der Kindheit. Frankfurt 1983.

Postman, N.: Keine Götter mehr. Das Ende der Erziehung. München 1997.

Rein, A. von (Hrsg.): Medienkompetenz als Schlüsselbegriff. Bad Heilbrunn 1996. 
Sander, U./Meister, D. (Hrsg.): Kinderalltag und Werbung. Zwischen Manipulation und Faszination. Neuwied 1997.

Schorb, B.: Medienalltag und Handeln. Medienpädagogik in Geschichte, Forschung und Praxis. Opladen 1995.

Stehr, N.: Arbeit, Eigentum Wissen, zur Theorie von Wissensgesellschaften. Frankfurt/M. 1994

Tietgens, H.: Von den Schlüsselqualifikationen zur Erschließungskompetenz. In: Petsch, H.-J./Tietgens, H. u.a. (Hrsg.): Allgemeinbildung und Computer, Bad Heilbrunn 1989, S. 34-43.

Treumann, K.P./Gartemann, S./Schnatmeyer, D./Röllecke, R.: Benjamin Blümchen, Bibi Blocksberg \& Co. Beliebte Kinder-Hörspielserien auf Cassette und CD. Bielefeld 1996.

Treumann, K.P./Volkmer, I.: Die Toncassette im kindlichen Medienalltag. In: Zentrum für Kindheitsund Jugendforschung (hrsg. von Melzer, W./Neubauer G./Sander, U./Volkmer, I.): Wandlungen der Kindheit. Theoretische Überlegungen zum Strukturwandel der Kindheit heute. Opladen 1993, S. 115-162.

Willke, H.: Supervision des Staates. Frankfurt/M. 1997. 